\title{
Enhancement of Speech Signal using Improved NLMS Algorithm
}

\author{
Rathnakara.S \\ Research Student, \\ JSS Research Foundation, \\ Mysore, India
}

\author{
V. Udayashankara, $\mathrm{PhD}$ \\ Research Guide, \\ JSS Research Foundation, \\ Mysore, India
}

\begin{abstract}
In this paper a modified robust variable step size algorithm is proposed and the performance of the proposed algorithm is compared with original robust variable step size (RVSS) algorithm. This leads to another derivative of Normalized Least mean square algorithm (NLMS). The performance of the proposed algorithm is measured with additive stationary and nonstationary Gaussian noise with original speech taken by standard IEEE sentence (SP23) of NOIZEUS data base. The output of proposed and RVSS algorithm are measured with excess mean square error (EMSE) in both stationary and non stationary environment. The results can be appreciated that the proposed algorithm gives improved result over RVSS algorithm and also the speed of convergence is maintained same as other NLMS algorithms
\end{abstract}

\section{General Terms}

Speech processing and noise estimation

\section{Keywords}

Speech enhancement, noise estimation, NLMS, RVSS, EMSE

\section{INTRODUCTION}

At the receiving end, most of the times the speech signal corrupted by some form of the noise components resulting in reducing the quality and intelligibility of speech. So the original speech becomes unpleasant to listen. The amount of unpleasant is depending upon the type of noise corrupted to the speech signal. The noise will be either stationary or nonstationary The quality and intelligibility of the speech signal which is corrupted with background noise can be improved by speech enhancement algorithms. The stationary noise can be easily estimated because their noise spectrum is constant. In case of non-stationary noise since the noise spectrum will be varying rapidly over time, noise spectrum needs to be estimated and uploaded continuously. Over many decades researchers are focused in this area and developed the different algorithms to remove the noise which is present along with the speech signal [1-7]. The adaptive filter is still better tool to suppress the background noise and enhancement of speech signal in noisy environment. In the basic least mean square (LMS) adaptive filter the step size remains constant in weight equation for all the input samples. [8]. The basic block diagram of adaptive noise canceller is shown in Fig1, Primary input $d(n)$ consists of the sum of desired signal $s(n)$ and uncorrelated noise $n_{0}(n)$. The noise $n_{0}(n)$ is uncorrelated with $s(n)$. The reference input $x(n)$ is another noise $n_{l}(n)$ which is correlated with $n_{0}(n)$ and uncorrelated with $s(n)$.

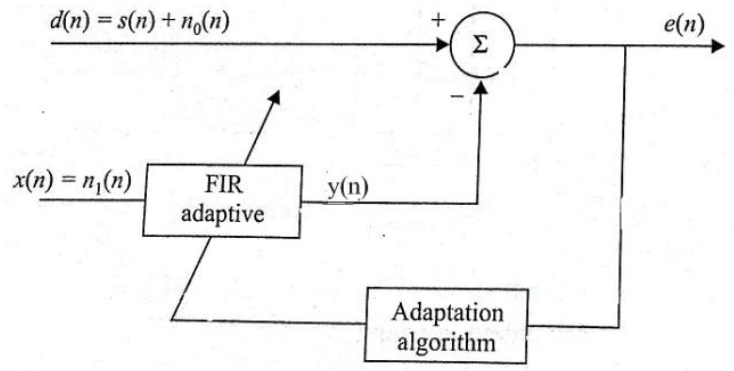

Fig 1. Adaptive noise canceller

\section{PROPOSED ALGORITHM}

The normalized LMS (NLMS) algorithm overcomes the fixed step size problem and step size varies from one iteration to the next, the weight vector of an adaptive filter change along with the input. The rate of convergence of NLMS algorithm is faster than that of LMS filter [9]. So the method of varying the step size is focused by many researchers and developed different forms of NLMS algorithms. In which robust variable step size (RVSS) algorithm [3] is also one among them. The proposed algorithm is modified RVSS algorithm, which shows improvement in decreasing EMSE and also minimizes signal distortion for both stationary and nonstationary noise

The output, error and weight equation of conventional NLMS algorithms are shown below.

The output; $y(n)=w(n) \cdot x^{T}(n)$

The error; $e(n)=d(n)-y(n)$

The weight equation:

$w(n+1)=w(n)+\frac{\mu}{\dot{\varepsilon}+\|x(n)\|^{2}} x(n) e(n)$

Where ' $\mathrm{w}$ ' is the adaptive filter vector weight and $\|x(n)\|^{2}$ is equal to $x(n)^{*} x^{T}(n)$, const $(\dot{\varepsilon})$ is used in the denominator to prevent the division by a very small number.

A modified form on NLMS algorithm introduced as robust variable step size (RVSS)[5] algorithm and the weight update equation is given by

$w(n+1)=w(n)+\frac{\mu\||| e L(n)\|^{2}}{\alpha\|e(n)\|^{2}+(1-\alpha)\|x(n)\|^{2}} x(n) e(n)$

Where $\|e(n)\|^{2}=\sum_{i=o}^{n-1}|e(n-i)|^{2}$

Where $\left\|e_{L}(n)\right\|^{2}=\sum_{i=o}^{L-1}|e(n-i)|^{2}$

Where ' $n$ ' represents the total number of samples and ' $L$ ' represents fixed number of samples selected on the basis to get optimum response. 
The proposed algorithm may be considered as modified RVSS algorithm which replaces the error vector for ' $n$ ' samples to fixed samples ' $L$ ', then the denominator term $\|e(n)\|^{2}$ $=|| e L(n) \|\left.\right|^{2}$

$w(n+1)=w(n)+\frac{\mu}{\alpha}+\frac{\mu\|e L(n)\|^{2}}{(1-\alpha)\|x(n)\|^{2}} x(n) e(n)$

Where $\frac{\mu}{\alpha}$ is very small and $\left(\frac{\mu}{1-\alpha}\right)$ is constant and represented by ' $\mu_{1}$ '. Hence above equation can rewrite as

$w(n+1)=w(n)+\frac{\mu_{1}\|e L(n)\|^{2}}{\|x(n)\|^{2}} x(n) e(n)$

Equation no (8) is similar to original NLMS algorithm but each step size is multiplied by\|eL $(n) \|^{2}$. Hence the tradeoff between NLMS and RVSS algorithm is multiplication of ' $\mu_{1}$ ' by a factor $\left.|| e L(n)\right|^{2}$ and this reduces computational complexity.

The performance of the adaptive noise canceller may be described in terms of the excess means square error (EMSE) or misadjustment ' $M$ ' [1-6]. The EMSE at the ${ }^{\text {th }}$ iteration is defined by

$\operatorname{EMSE}(n)=\frac{1}{L} \sum_{j=0}^{L-1}|e 1(n-J)|^{2}$

Where $e l(n)=e(n)-s(n)$ is the excess residual error.

The steady state excess mean square error (EMSEss) is defined by

$$
\text { EMSEss }=\frac{1}{\mathrm{~K}-\mathrm{P}} \sum_{\mathrm{n}=\mathrm{P}}^{\mathrm{K}-1} \operatorname{EMSE}(n)
$$

Where $\mathrm{K}$ is the total number of samples of the speech signal and $\mathrm{P}$ is the number of samples after which the algorithm reaches steady state.

The misadjustment is given by

$\mathrm{M}=\frac{\text { EMSEsS }}{\text { MSEmin }}$

Where MSE $\min =\frac{1}{\mathrm{~K}-\mathrm{P}} \sum_{n=\mathrm{P}}^{\mathrm{K}-1}|s(n)|^{2}$

\section{SIMULATED RESULTS}

The simulations are carried with sp23 IEEE standard data base of male voice saying" stop whistling and watch the boys march" the original signal has 21209 samples. The simulations are carried with $\mathrm{K}=21209, \mathrm{~N}=10$ (length of the filter), $\mathrm{L}=200 \mathrm{P}=1, \alpha=0.7$ and $\mu=0.1$

\subsection{Stationary noise:}

The performance of the algorithms are computed by adding white Gaussian noise of zero mean with three different variance levels at $0.001,0.01$ and 0.1 . Fig 2 illustrates the performance of the RVSS algorithm for stationary noise corrupting the speech signal with variance $\left(\sigma^{2}\right)$ of 0.01 . From top to bottom that figure shows input signal, noise, signal+noise, output and minimum mean square error. The same for proposed algorithm is shown in Fig 3. The comparison of EMSEss in $\mathrm{dB}$ for both the algorithms is shown in Fig4. Table 1 illustrates the simulation results of stationary noise for both RVSS and proposed algorithm at different levels of variance. Which is clearly demonstrates that the EMSEss decreases in proposed algorithm compared with RVSS for different noise levels and also proposed algorithm maintains almost constant EMSEss for increasing variance level in the noise.

\subsection{NonStationary noise:}

For nonstationary case noise is assumed as white Gaussian noise with zero mean with a variance that linearly increases from minimum of 0.001 with different maximum variance levels at $0.0011,0.0101$, and 0.1001. Fig 5 illustrates the performance of RVSS algorithm for nonstationary noise corrupting the speech signal with $\sigma^{2} \min =0.001$ \& $\sigma^{2} \max =0.0101$. From top to bottom that figure shows the input signal, noise, signal+noise, output and minimum mean square error. The same for proposed algorithm is shown in Fig 6. Fig7 represent the comparison of EMSEss in $\mathrm{dB}$ for both the algorithms. Table 2 illustrates the simulation results for nonstationary noise for both RVSS and proposed algorithm. Similar to stationary noise the EMSEss decreases in proposed algorithm compare with RVSS for different noise levels and also proposed algorithm maintains almost constant EMSEss for increasing variance level in the noise.
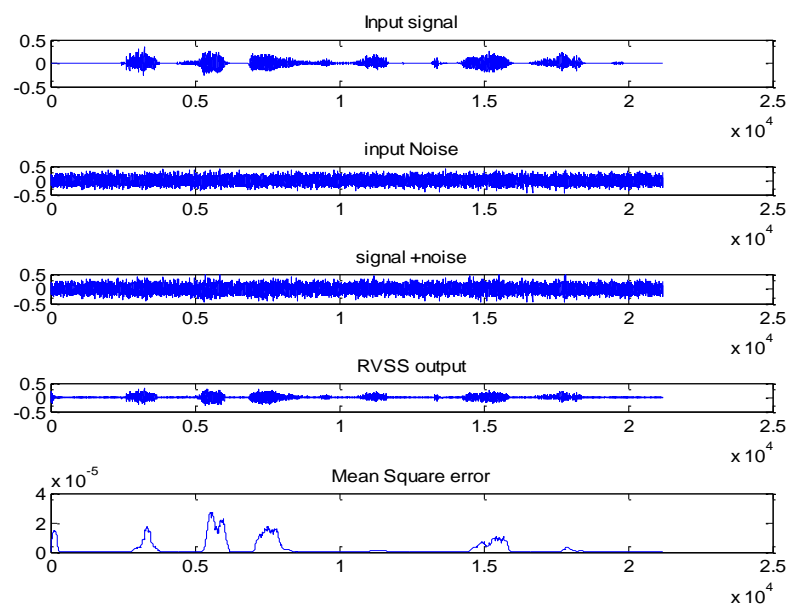

Fig 2. From top to bottom, the original speech, the noise, signal+noise, noise cancelled speech and excessive mean square error of RVSS algorithm for stationary noise

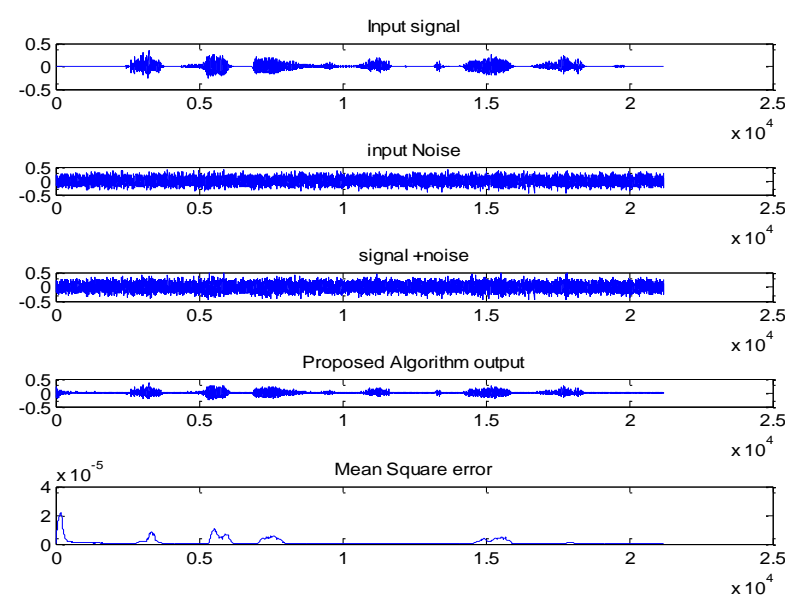

Fig 3. From top to bottom, the original speech, the noise, signal+noise, noise cancelled speech and excessive mean square error of proposed algorithm for stationary noise 

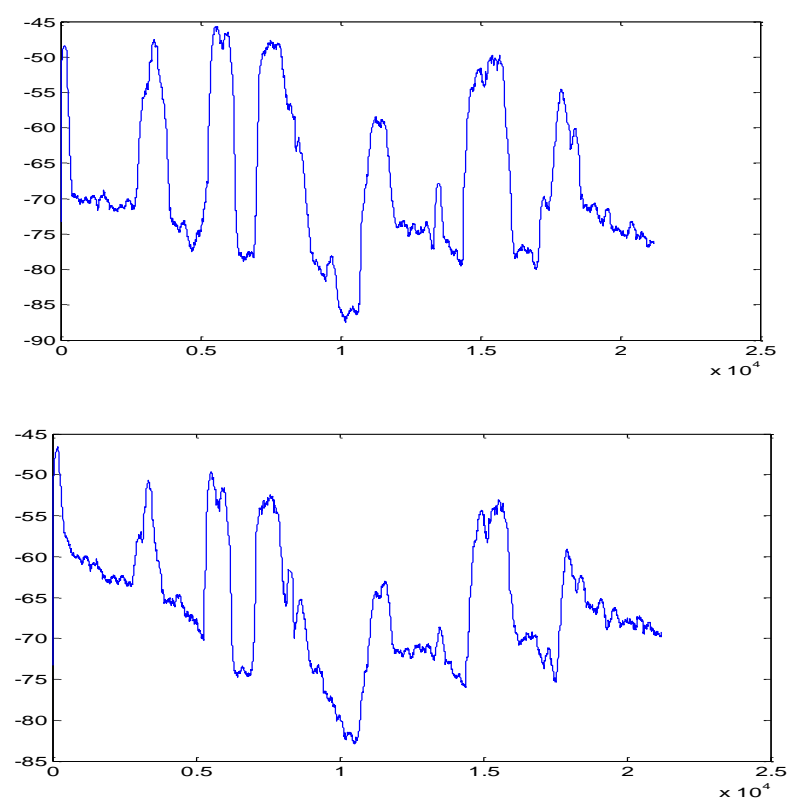

Fig 4. Top to bottom, excessive mean square error of RVSS and proposed algorithm in $\mathrm{dB}$ for stationary noise

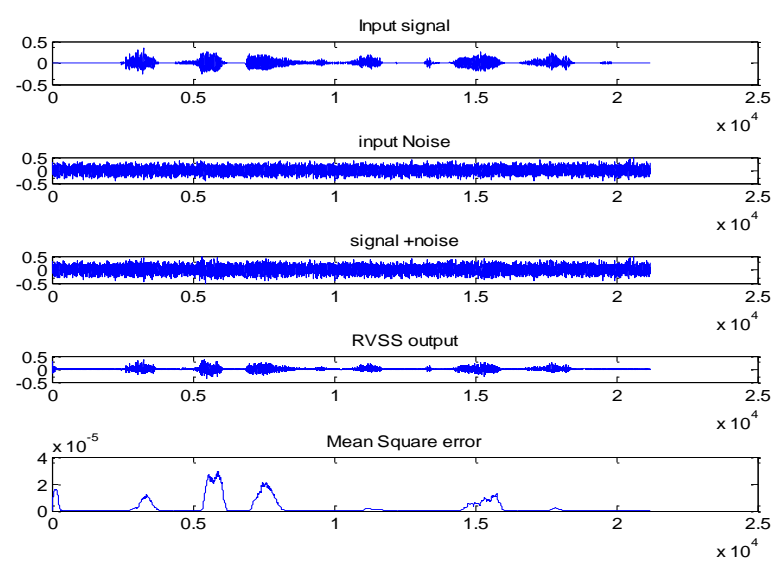

Fig 5. From top to bottom, the original speech, the noise, signal+noise, noise cancelled speech and excessive mean square error of RVSS algorithm for nonstationary $\operatorname{noise}\left(\sigma^{2} \max =\mathbf{0 . 0 1 0 1}\right.$,

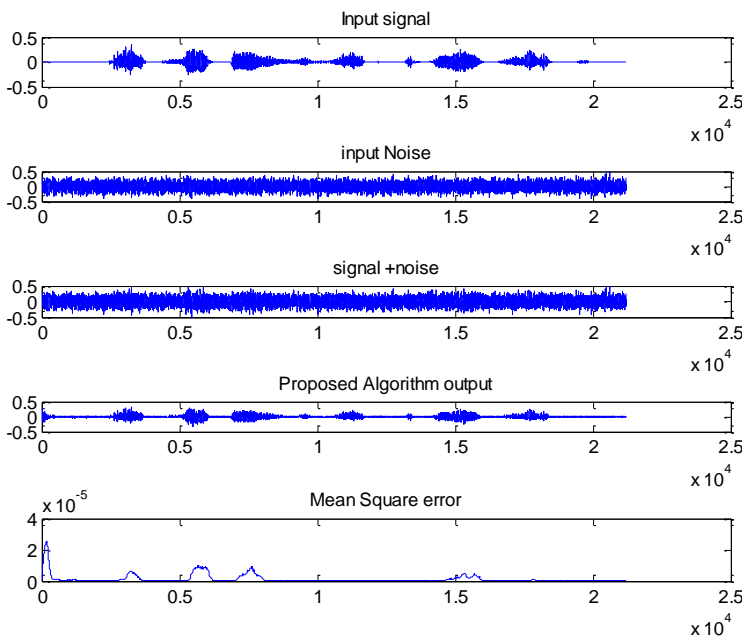

Fig 6. From top to bottom, the original speech, the noise, signal+noise, noise cancelled speech and excessive mean square error of proposed algorithm for nonstationary noise $\left(\sigma^{2} \max =0.0101\right)$
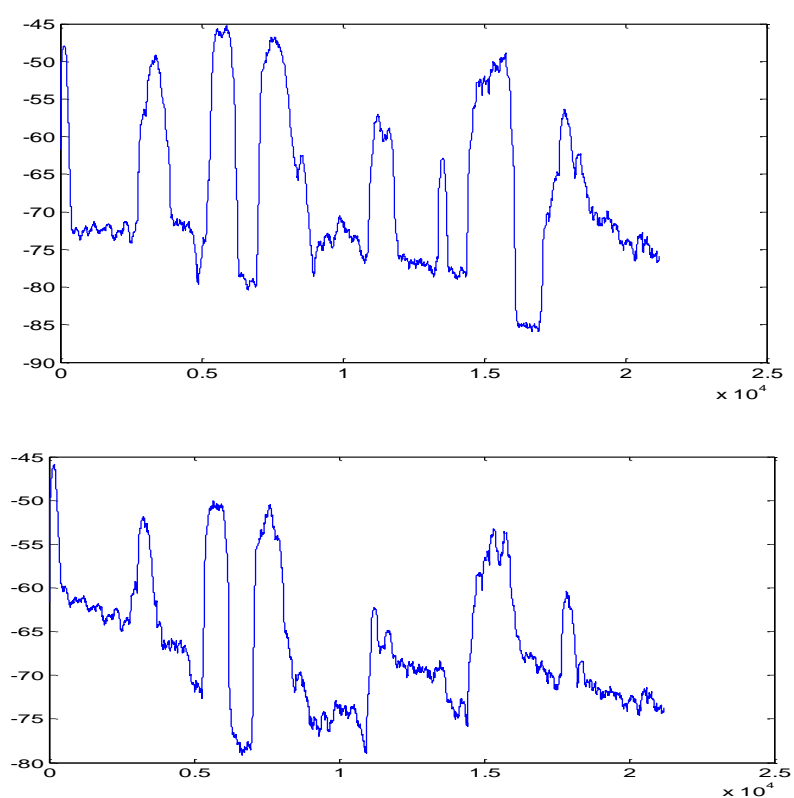

Fig 7. Top to bottom, the excessive mean square error of RVSS and proposed algorithm in dB for nonstationary noise

Table1: Comparison of EMSEss and Misadjustment for Stationary noise (Mean=0)

\begin{tabular}{|l|l|l|l|l|l|l|}
\hline \multicolumn{2}{|l|}{$\sigma^{2}=\mathbf{0 . 0 0 1}$} & \multicolumn{2}{l|}{$\sigma^{2}=\mathbf{0 . 0 1}$} & \multicolumn{2}{l|}{$\sigma^{2}=\mathbf{0 . 1}$} \\
\hline Algorithm & M & EMSEss (dB) & M & EMSE (dB) & M & $\begin{array}{l}\text { EMSEss } \\
(\mathrm{dB})\end{array}$ \\
\hline RVSS & 0.0601 & -60.17 & 0.1395 & -56.52 & 0.2668 & -53.70 \\
\hline $\begin{array}{l}\text { Proposed } \\
\text { Algorithm }\end{array}$ & 0.0606 & -60.13 & 0.0705 & -59.48 & 0.0954 & -58.17 \\
\hline
\end{tabular}


Table2: Comparison of EMSEss and Misadjustment for Nonstationary noise (Mean=0; $\left.\sigma^{2} \mathbf{m i n}=0.0001\right)$

\begin{tabular}{|c|c|c|c|c|c|c|}
\hline & \multicolumn{2}{|c|}{$\sigma^{2} \max =0.0011$} & \multicolumn{2}{|c|}{$\sigma^{2} \max =0.0101$} & \multicolumn{2}{|c|}{$\sigma^{2} \max =0.1001$} \\
\hline Algorithm & $\mathrm{M}$ & EMSEss (dB) & $\mathrm{M}$ & EMSEss (dB) & $\mathrm{M}$ & $\begin{array}{l}\text { EMSEss } \\
(\mathrm{dB})\end{array}$ \\
\hline RVSS & 0.0746 & -59.23 & 0.1480 & -56.26 & 0.2588 & -53.20 \\
\hline $\begin{array}{l}\text { Proposed } \\
\text { Algorithm }\end{array}$ & 0.0628 & -59.99 & 0.0726 & -59.36 & 0.0991 & -58.00 \\
\hline
\end{tabular}

\section{CONCLUSION}

In this paper a new normalized step size algorithm is proposed by modifying RVSS algorithm. The results are demonstrating the performance of the RVSS and modified algorithms for both stationary and nonstationary noises at different levels. The proposed algorithm reduces misadjustment and steady state minimum mean square error (EMSEss) and also increases tracking capability of the input compare to original RVSS algorithm. The performance of the algorithm can further improve if length of the fixed samples (L) is automated. The proposed algorithm can be used in automatic noise canceller, echo removal and noise cancellation in digital hearing aids

\section{REFERENCES}

[1] Alexander D.Poularikas, Zayed M.Ramadan "Adaptive filtering primer with MATLAB CRC press

[2] Zayed Ramadan, \&Alexander Poularikas ,Performance Analysis of a New Variable Step-Size LMS Algorithm with Error Nonlinearities IEEE 2004 pp384-388

[3] Zayed Ramadan and Alexander Poularikas A Robust Variable Step-Size LMS Algorithm Using Error-Data Normalization IEEE 2005 PP219-224
[4] Zayed Ramadan and Alexander Poularikas A Variable Step-Size Adaptive Noise Canceller Using Signal to Noise Ratio as the Controlling Factor IEEE2004 PP 456461

[5] Zayed Ramadan and Alexander Poularikas An Adaptive Noise Canceller Using Error Nonlinearities in the LMS Adaptation IEEE 2004 pp3-8

[6] Joonwan Kim \&Alexander D. Poularikas "Performance analysis of the Adjusted step size NLMS algorithm IEEE 2004 pp 467-571

[7] Ma Shengqian, Xu Guowei, Ma Zhifeng, Wei Shuping, Fan Manhong "Research on adaptive noise canceller of an improvement LMS algoritnm 2011 IEEE pp16111614

[8] V.Udayashankara " Modern digital signal processing" second edition PHI

[9] Simon Haykin “ Adaptive Filter Theory" Fourth edition pearson education 